\title{
A P2P-Based Approach to Cross-Domain Network and Service Management
}

\author{
Adriano Fiorese $^{1,2}$, Paulo Simões ${ }^{1}$, and Fernando Boavida ${ }^{1}$ \\ ${ }^{1}$ Centre for Informatics and Systems of the University of Coimbra - CISUC \\ Department of Informatics Engineering - DEI \\ University of Coimbra - UC \\ fiorese@dei.uc.pt, psimoes@dei.uc.pt, boavida@dei.uc.pt \\ 2 Department of Computer Science - DCC \\ University of the State of Santa Catarina - UDESC \\ 890233-100 Joinville, SC, Brazil \\ fiorese@joinville.udesc.br
}

\begin{abstract}
Cross-domain network and service management is still a challenging task. Most network management solutions that exist today are only applicable to single, homogeneous domains. Due to their distribution and technology-independence, P2P overlays have very good potential to boost inter-domain network and service management. The proposed work intends to explore this potential in order to develop efficient, trusted and secure cross-domain management, with benefits in terms of performance, resilience and support for autonomic operation. This paper explains the main challenges in this respect, discusses the basic approach to be taken and identifies the contributions.
\end{abstract}

Keywords: Network and Service Management, P2P, Overlays.

\section{Problem Description}

Efficient and effective solutions to network and services management are, more than ever, an unfulfilled promise. As networks and domains get larger and their inter-relations get more complex, the lack of solutions for global, end-to-end network management becomes apparent. On the other hand, users are requesting (and paying) services that require monitoring and control in order to guarantee a certain quality of experience, and do not care if the service spans several domains or requires complex low-level resource management.

The main obstacles for network management are administrative domain frontiers. Relatively good and stable solutions exist for intra-domain management, but they are not feasible at inter-domain level due to two main reasons: 1) administrative barriers imposed by domain owners; 2) several low-level management operations cannot be performed and/or are not efficient at this level (e.g., simple SNMP-based 12 solutions, or even Management by Delegation (MbD) 3. solutions). 
In this respect, some researchers have begun to explore P2P-based systems for network management $[4,5,6]$. The potential of $\mathrm{P} 2 \mathrm{P}$ overlays in the network management area is very good, as they lead to several immediate benefits: independence in relation to underlying technologies and providers; domain transparency; very good level of abstraction; and, last but not least, easy support of a user-oriented quality of experience view of the services.

The work proposed and presented in this paper addresses and explores the use of P2P-based overlays for network and service management in cross-domain environments.

\section{Challenges}

The main challenges of the proposed work are summarized in the following:

- Efficient cross-domain management;

- Provision of a trusted and secure solution;

- Distributed support for configuration operations;

- Improved performance when compared to traditional network management approaches;

- Enhanced resilience;

- Support for autonomic management, comprising intelligent communication, network control and management paradigms.

Current network management frameworks were developed for intra-domain environments, in which managing entities and managed entities trust each other and interact on a cooperation basis. In this context, all management information is available for managers, with various levels of detail. This is, of course, not the case when several administrative domains exist. In general, management information concerning one domain is hidden from entities in other domains, or is highly filtered. The use of a peer-to-peer overlay formed by nodes belonging to different administrative domains offers network managers the possibility of cooperatively managing these different domains. Management services provided by the overlay nodes are not bounded by domain borders, as these borders do not exist in the overlay. All overlay nodes cooperate, providing management services to each other.

One important challenge is that of trust and security. Appropriate mechanisms must be in place for ensuring that management operations performed in the overlay are carried out with the necessary assurance in terms of authentication and authorization. In addition, these operations must be translated into proper low-level inter-domain management operations when appropriate, filtering sensitive information and guaranteeing the necessary trust level.

Another challenge relates to configuration operations. Traditional solutions for network resources configuration are based on centralized configuration data repositories and it is part of a centralized approach. Large scale management configuration updates, however, can push servers and links to the limit. Additionally, centralized approaches lead to single points of failure and pose scalability 
problems. In this respect, $\mathrm{P} 2 \mathrm{P}$ approaches open a range of possibilities that will be explored in the context of this work, including increased data configuration resilience and bandwidth savings.

Adequate performance of the network management operations is also a challenge. In the case of a centralized large scale management operation, the NMS may have to trigger a high number of messages which will cross the entire network domain and will reach managed elements one by one. A mix of management by delegation and P2P can achieve better performance. For instance, once a peer belonging to the management overlay receives a command for an update operation, it can perform the operation quicker, as it lies nearer to the network element than the central NMS.

Another motivation for the use of a P2P overlay for network management is the possibility of keeping network partitions managed in the event of a partitioning on the whole network due to failure of network elements or links. In a centralized network management approach, when a network partition occurs, just the partitions where the network managers reside remain managed. However when a self-organizing P2P overlay is used, the partitions themselves can elect nodes to take up management responsibility and keep things working.

Global inter-domain scope, trust and security, distributed operation, efficient load balancing and enhanced resilience are key aspects closely related to $\mathrm{P} 2 \mathrm{P}$ systems that can be explored in order to pursue autonomic management, comprising intelligent communication, network control and management paradigms. The P2P approach can lead to smart network and service management entities that are capable of automatically configuring and organizing themselves.

\section{Approach}

Our approach consists of building a P2P-based network management framework to cope with the identified challenges. It will explore the cross-domain ability of $\mathrm{P} 2 \mathrm{P}$ overlays to manage network resources and services. The proposed approach builds on and goes beyond of related work 456. In our approach every peer in the network management overlay will be able to execute some management functions. These management functions/services will be published to network administrators, and will be made available to management tools. To do that, the peers in the overlay will advertise their services to one or more entities which will aggregate these advertisements. The component responsible for that is called Aggregation Service (see Fig. 1), and it will be composed of super-peers in the management overlay that will collect the management services advertised by the peers in the overlay. It is expected that this component can improve the search speed for specialized management services. These advertised management services will be looked up by high level managers in order to know which peers provide the services and which management interfaces can be used. Specialized management service providers can join the overlay in an non-deterministic way, offering their services. New management services can be deployed in the overlay in a natural way, just starting up the software. 


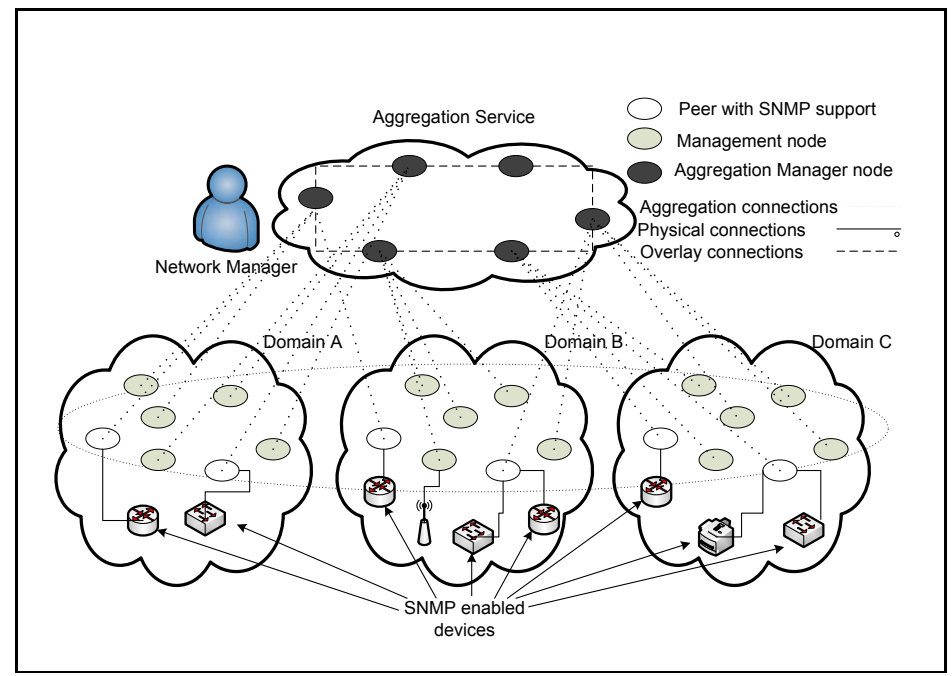

Fig. 1.

\section{Expected Contributions}

- The proposal, implementation and evaluation of a P2P-based network and service management system;

- A novel cross-domain management approach, adequately dealing with the trust and security issues that are the main obstacles to the deployment of management solutions spanning several administrative domains;

- An approach to distributed configuration operations, enhancing performance and resilience;

- The development of a set of mechanisms which can support autonomic management, in inter-domain environments.

\section{References}

1. Case, J.D., Fedor, M., Schoffstall, M., Davin, J.: Rfc1157 - simple network management protocol (SNMP) (1990)

2. Case, J.D., Mundy, R., Partain, D., Stewart, B.: Rfc3410 - introduction and applicability statements for internet standard management framework (2002)

3. Goldszmidt, G., Yemini, Y.: Distributed management by delegation. In: Proceedings of the 15th ICDCS, pp. 333-340 (1995)

4. Leitner, M., Leitner, P., Zach, M., Collins, S., Fahy, C.: Fault management based on peer-to-peer paradigms; a case study report from the celtic project madeira. In: 10th Integrated Network Management (IM), May 2007, pp. 697-700 (2007)

5. Granville, L.Z., da Rosa, D.M., Panisson, A., Melchiors, C., Almeida, M.J.B., Tarouco, L.M.R.: Managing computer networks using peer-to-peer technologies. IEEE Communications Magazine 43(10), 62-68 (2005)

6. State, R., Festor, O.: A management platform over a peer-to-peer service infrastructure. In: 10th International Conference on Telecommunications (ICT 2003), vol. 1, pp. 124-131 (2003) 